\title{
Self-Organized Authentication Architecture for Mobile Ad-hoc Networks
}

\author{
P. Caballero-Gil*, C. Caballero-Gil ${ }^{\dagger}, J^{*}$ Molina-Gil ${ }^{\dagger}$, C. Hernández-Goya* \\ ${ }^{*}$ Dept. Statistics, Operations Research and Computing. University of La Laguna. 38271 La Laguna. Tenerife. \\ Spain. E-mail: pcaballe,mchgoya@ull.es \\ ${ }^{\dagger}$ Dept. Informatics and Systems. University of Las Palmas de Gran Canaria. 35017 Las Palmas de Gran Canaria. \\ Gran Canaria. Spain. E-mail: candido.caballero,jezabelmiriam@gmail.com
}

\begin{abstract}
This work proposes a new architecture, called Global Authentication Scheme for Mobile Ad-hoc Networks (GASMAN), for fully distributed and self-organized authentication. In this paper apart from describing all the GASMAN components, special emphasis is placed on proving that it fulfils every requirement of a secure distributed authentication scheme, including limited physical protection of broadcast medium, frequent route changes caused by mobility, lack of structured hierarchy, etc. Furthermore, an extensive analysis through simulation experiments in different scenarios is described and discussed.
\end{abstract}

Keywords. Authentication, Access Control, MANETs

\section{INTRODUCTION}

Network security consists of several services such as authentication, confidentiality, integrity, non-repudiation, and access control. Among these facilities, authentication, which ensures the true identities of nodes, is the most fundamental one because other services depend fully on the sure authentication of communication entities.

Mobile Ad-hoc NETworks (MANETs) are autonomous networks formed by mobile nodes that are free to move at will. These networks have received increasing interest in the last years, partly owing to their potential applicability to myriad applications, ranging from small, static networks that are constrained by power sources, to large-scale, mobile and highly dynamic networks. Conventional wired networks mainly use a globally trusted Certificate Authority (CA) for solving the authentication problem. However, authentication in MANETs is undoubtedly much more difficult to solve due to several reasons such as the absence of a fixed infrastructure and centralized management, the dynamic nature of the nodes, the limited wireless range of nodes, the dynamic topology, frequent link failures and possible transmission errors [1] [12]. Also, since all the nodes must collaborate to forward data, the wireless channel is prone to active and passive attacks by malicious nodes, such as Denial of Service (DoS), eavesdropping, spoofing, etc.

One of the most elementary approaches to authentication in MANETs found in the bibliography uses a Trusted Third Party (TTP) to guarantee the validity of all nodes identities so that every node who wants to join the network has to get a certificate from the TTP. A second identification paradigm that has been used in wireless ad-hoc networks is the notion of chain of trust [8]. A third typical solution is locationlimited authentication, which is based on the fact that most adhoc networks exist in small areas and physical authentication may be carried out between nodes that are close to each other. The special nature of ad-hoc networks, where most applications are collaborative and group-based, suggests that such traditional approaches to node identification may not be always appropriate. As an example of this fact, we have that different authentication protocols have been recently proposed for ad-hoc networks but all of them have some drawback. For instance, the work [7] is based on the RSA signature, which conducts to the problem of public key certification. Another recent example is the paper [11], which provides a solution that works well just for short-lived MANETs. Thus, the design of a new scheme that fulfils all the requirements for this type of networks continues being considered an open question.

Here we propose a new architecture for authentication in ad-hoc networks called Global Authentication Scheme for Mobile Ad-hoc Networks (GASMAN), which is based on the established cryptographic paradigm of Zero-Knowledge Proofs (ZKPs). Since the information sent while its execution does not convey any secret related to the authentication process, ZKPs provide an elegant and fault-tolerant solution to node authentication in MANETs. Furthermore, the GASMAN has other three beneficial properties. Firstly, it has scalability because centralized elements, such as CA servers, are not required. Secondly, availability is guaranteed through the insertion, deletion and access control procedures. Finally, our architecture assures strong authentication to any legitimate node willing to join the network by using the zeroknowledge proof implemented in the access control algorithm. The GASMAN algorithms jointly with mobility help to reduce the time necessary for nodes to join and access the network in a timely manner. Summing up, the main features of the proposed protocol are the adaptation to the varying topology of the network, the open availability of broadcast transmissions and the strong access control.

Up to now, very few publications have mentioned the proposal of authentication systems for ad-hoc networks using ZKPs [2] [13], and none of them has dealt with the related problem of topology changes in the network. A recent ZKPbased hierarchical proposal for MANETs related with the one 
proposed here was described in [4], where two different security levels were defined through the use of a hard-on-average graph problem, and no topology changes were considered.

This work is organized as follows. The following section provides an overview of the proposal, some general aspects of the proposal and the notation used in its description. Specific details about the five principal elements of the architecture, i.e., network initialization, node insertion, access control, proofs of life and node deletion are gathered in Section III. The assumptions required by GASMAN and an analysis of its security are commented in Section IV. A performance analysis developed under NS-2 is provided in Section V. Finally, some conclusions and open questions complete the paper.

\section{BASICS AND NOTATION}

The proposed protocol was designed as an authentication scheme for group membership because when a node wants to be part of the network, it has to be previously authorized by a legitimate node. According to the authors of [10], in any group member authentication protocol it is necessary to provide robust methods to insert and to delete nodes, as well as to allow the access only for legitimate members of the group. For that reason, not only the ZKP used for access control is described, but also the update procedures associated to insertions and deletions are carefully defined. For instance, the procedure to delete nodes is only initiated once a node has been disconnected of the network for too long. The period of time after which the node is deleted is a parameter $(T)$ of the system here presented.

The access control described below is based on the general scheme of Zero-Knowledge Proof introduced in [3], when using the Hamiltonian Cycle Problem (HCP). A Hamiltonian cycle in a graph is a cycle that visits each vertex exactly once and returns to the starting vertex. Determining whether such cycles exist in a graph defines the Hamiltonian Cycle Problem, which is NP-complete. Such a problem was chosen for our design mainly due to the low cost of the operations associated to the update of a solution. This is an important characteristic since in a highly dynamic setting such as MANETs these operations will be developed frequently. Anyway, there should be pointed out that similar schemes based on different NPcomplete graph problems might be described. The only feature demanded to the problems chosen is that the solutions may be easily updated when small changes occur in the network. This is just the case of the Vertex Cover, Independent Set or Clique Problems, for instance.

One of the key points to assure the correct operation of GASMAN is the use of a chat application through broadcast that makes it possible for legitimate on-line nodes to send a message to all on-line users. Such an application allows publishing all the information associated to the update of the network. Although secrecy is not necessary for chat messages, because they are not more than gibberish for illegitimate nodes, it is required that only the on-line legitimate nodes execute the chat application.
The information received through the chat application during an interval of time must be stored by each on-line node in a FIFO queue. Such data should be stored by each on-line node, allowing in this way the updating of the authentication information not only to it but also to all the off-line legitimate nodes whose access will be granted. Such a period is an essential parameter in the system because it states both the maximum off-line time allowed for any legitimate node, and the frequency of broadcasting the proofs of life. Consequently, such a parameter should be previously agreed among all the legitimate nodes of the network.

A generic life-cycle of a MANET has three major phases that are described below (see figure 1):

\section{Initialization:}

Each initial member in the original network will be securely provided, either off-line or on-line, with a secret piece of information. The knowledge of the secret network key will be used during access control in order to prove the node's eligibility for accessing to the protected resources or to offer service to the network. After completing this stage, the legitimate nodes are ready to actively participate in the network.

\section{Access Control:}

The access control process allows a legitimate node to prove its network membership to an on-line node. These legitimate nodes must demonstrate knowledge of the secret network key by using a challengeresponse scheme.

\section{On-line Session:}

Once the legitimate node reaches an on-line state in the network, it gets full access to the protected resources such as the chat application. At the same time, it may offer services such as the insertion of new nodes. There should be taken into account that the secret network key will be updated according to the network evolution. Hence, if a node is off-line for too long, its secret key will expire. In such a case, the legitimate node would have to be re-inserted by an on-line legitimate node.

Since in our proposal the secrecy of the network key is provided by the difficulty of the HCP, the number of on-line legitimate nodes is a crucial parameter. In consequence, as soon as the number of on-line legitimate nodes becomes too small (when comparing it with certain threshold parameter), the network termination is carried out and therefore, the lifecycle of the network ends.

Probably, the most remarkable aspect in our proposal is that no meaningful information may be stolen even if an adversary is able to reads the whole information published through the chat application, or even if it eavesdrops the information exchanged between a legitimate prover and verifier at the time of executing the access control protocol.

In the following, the basic notation used throughout the proposal is explained.

- $G_{t}=\left(V_{t}, E_{t}\right)$ denotes the undirected graph used at stage 


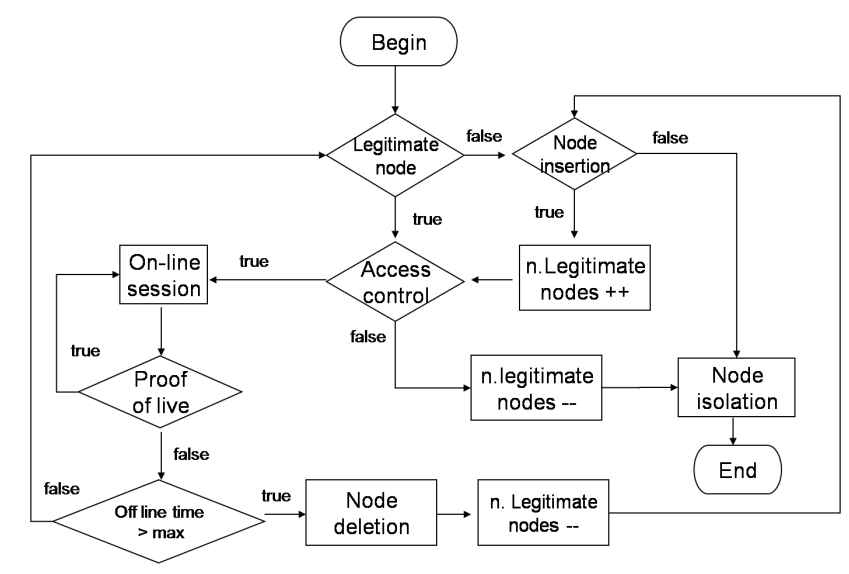

Fig. 1. Node Life-Cycle

$t$ of the network life-cycle.

- $v_{i} \in V_{t}$ represents both a vertex of the graph and a legitimate node.

- $n=\left|V_{t}\right|$ is the order of $G_{t}$, which coincides with the number of legitimate nodes.

- $N_{G_{t}}\left(v_{i}\right)$ denotes the neighbours of node $v_{i}$ in the graph $G_{t}$.

- $\Pi\left(V_{t}\right)$ represents a random permutation over the vertex set $V_{t}$

- $\Pi\left(G_{t}\right)$ denotes the graph isomorphic to $G_{t}$ built after applying permutation $\Pi\left(V_{t}\right)$.

- $c \in_{r} C$ indicates that an element $c$ is chosen at random with uniform distribution from a set $C$.

- $H C_{t}$ designates the Hamiltonian cycle used at stage $t$.

- $\Pi\left(H C_{t}\right)$ represents the Hamiltonian cycle $H C_{t}$ in the graph $\Pi\left(G_{t}\right)$.

- $N_{H C_{t}}\left(v_{i}\right)$ denotes the neighbours of node $v_{i}$ in the Hamiltonian cycle $H C_{t}$.

- $S$ and $A$ stand for the supplicant and the authenticator, respectively. This notation is used both while an insertion phase and the execution of a ZKP-based access control are carried out.

- $S \rightleftharpoons A$ symbolizes when node $S$ contacts $A$.

- $A \leftrightarrow S$ : information means that $A$ and $S$ agree on information

- $A \stackrel{s}{\rightarrow} S$ : information means that $A$ sends information to $S$ through a secure channel.

- $A \stackrel{o}{\rightarrow} S:$ information means that $A$ sends information to $S$ through an open channel.

- $A \stackrel{b}{\rightarrow}$ network : information represents when $A$ broadcasts information to all on-line legitimate nodes.

- $A \stackrel{b}{\leftrightarrow}$ network : information represents a two-step procedure where $A$ broadcasts information to all online legitimate nodes of the network, and receives their answers.

- $h$ stands for a public hash function.

- $T$ denotes the threshold period that a legitimate nodes may be off-line without been excluded of the network.

\section{GASMAN DESCRIPTION}

This section contains the description of the procedures that form part of the GASMAN architecture, including all the specific details about network initialization, node insertion, access control, proofs of life and node deletion.

\section{A. Network Initialization}

The proposed protocol requires the definition of an initialization phase where the secret information associated to the process of identification is generated and distributed within the initial network. This initialization phase consists in the definition of the graph used for the development of the protocol. Such a graph should be generated with the participation of all the original members of the network. Furthermore, the initialization phase also implies the shared generation by the initial legitimate members of the network of a solution to the HCP in such a graph.

In our proposal, as in trust graphs [9], the graph vertexes set corresponds to the set of nodes in the actual network during its whole life-cycle. Consequently, the initialization process starts from a set $V_{0}$ of $n$ vertexes corresponding to the nodes of the initial network. Hence, each vertex sub-index may be used as ID (IDentification) for the corresponding node. The first step of the initialization process consists of generating cooperatively and secretly a random permutation $\Pi$ of such a set. Once this generation is completed, each legitimate node should know a Hamiltonian cycle $H C_{0}$ corresponding exactly to such a permutation. Finally, the partial graph formed by the edges corresponding to such a Hamiltonian cycle $\mathrm{HC}_{0}$, is completed by adding $n$ groups of $\frac{2 m}{n}$ edges, producing the initial edge set $E_{0}$. Here, $m$ stands for the number of edges that the initial graph will have after the initialization stage. Each one of these $n$ groups of edges will be generated by $v_{i}, i=1,2, \ldots, n$ according to the following restrictions: they must have $v_{i}$ as one of its vertexes, while the other one will be randomly generated. Note that the size $\frac{2 m}{n}$ of those edge subsets must be large enough so that the size of the resulting edge set $\left|E_{0}\right|=m$ guarantees the difficulty of the HCP in the graph $G_{0}$. 
Initialization Algorithm

Input: $V_{0}$, with $\left|V_{0}\right|=n$.

1. The $n$ nodes of the network generate cooperatively, secretly and randomly the cycle $H C_{0}=\Pi\left(V_{0}\right)$.

2. $\forall v_{i} \in V_{0}, v_{i}$ builds the set

$$
N_{G_{0}}(i)=\left\{\left\{v_{j} \in_{r} V_{0}\right\} \cup N_{H C_{0}}(i)\right\}
$$

$$
\text { with }\left|N_{G_{0}}(i)\right|=\frac{2 m}{n} \text {. }
$$

3. $\forall v_{i} \in V_{0}: v_{i} \stackrel{b}{\rightarrow}$ network $: N_{G_{0}}(i)$.

4. $\forall v_{i} \in V_{0}: v_{i}$ merges:

$$
E_{0}=\bigcup_{i=1,2, \ldots, n}\left\{\left(v_{i}, v_{j}\right): v_{j} \in N_{G_{0}}(i)\right\} .
$$

Output: $G_{0}=\left(V_{0}, E_{0}\right)$, with $\left|E_{0}\right|=m$.

Once the creation of the initial instance of the problem has been carried out through the contribution of all the nodes of the network, each node will know a Hamiltonian cycle in the resulting $\frac{2 m}{n}$-regular graph. From then on, each time a new user $S$ wants to become a member of the network, it has to contact a legitimate member $A$ in order to follow the insertion procedure explained in the following section.

\section{B. Node Insertion}

Let us suppose that we are at stage $t$ of the network lifecycle when a user $S$ contacts a legitimate member $A$ of the network to become a member of the network. Once $S$ has convinced $A$ to accept its membership in, the first step that $A$ should carry out is to assign $S$ the lowest vertex number $v_{i}$ not assigned so fat in the vertex set $V_{t}$. Afterwards, $A$ should broadcast such an assignment to all on-line legitimate nodes in order to prevent another simultaneous insertion with the same identifier. If $A$ receives less than $n / 2$ answers to the previous message, she stops the insertion procedure because the number of nodes that are aware of the insertion is not large enough. Otherwise, $A$ develops the corresponding update of the secret Hamiltonian cycle $H C_{t}$ by selecting at random two neighbour vertexes $v_{j}$ and $v_{k}$ in order to insert the new node $v_{i}$ between them. Additionally, $A$ chooses at random a subset of $\frac{2 m}{n}-2$ nodes in $V_{t}$ such that none of them is its neighbour in $H C_{t}$. Finally, $A$ broadcasts the set of neighbours $N_{G_{t+1}}\left(v_{i}\right)$ of $S$ in the new graph $G_{t+1}$.

Each time a node receives a graph update, it should secretly modify the corresponding Hamiltonian cycle. In order to achieve it, it uses the information provided to identify the unique position (according to the new edge set $E_{t+1}$ ) in the cycle where the new node can be inserted. In this way, it will be able to easily update the secret network key by simply inserting the vertex $v_{i}$ between the vertexes $v_{j}$ and $v_{k}$. At the same time, the authenticator node $A$ must send the supplicant node $S$ both the graph $G_{t+1}$ (deploying an open channel), and the Hamiltonian cycle $\mathrm{HC}_{t+1}$ (through a secure channel).

Insertion Algorithm

Input: At stage $t$ a supplicant node $S$ wants to become a member of the network.

1. $S \rightleftharpoons A$.
2. Node $S$ convinces node $A$ to accept its membership in the network.

2. $\quad A$ assigns $S$ the identifier $v_{i}$ such that $i=\min \{l$ : $\left.v_{l} \notin V_{t}\right\}$

3. $\quad A \stackrel{b}{\leftrightarrow}$ network : $v_{i}$

3.1 If $A$ receives less than $n / 2$ answers, she stops the insertion procedure.

3.2 Otherwise:

3.2.1 A chooses: $\left(v_{j}, v_{k}\right): v_{j} \in_{r} V_{t}, v_{k} \in_{r} N_{C H_{t}}\left(v_{j}\right)$

3.2.2 $A$ chooses at random:

$N_{G_{t+1}}\left(v_{i}\right)=\left\{v_{j}, v_{k}\right\} \cup\left\{w_{1}, w_{2}, \ldots, w_{\frac{2 m}{n}-2}\right\}$ such that $N_{G_{t+1}}\left(v_{i}\right) \subseteq V_{t} \wedge \forall w_{l_{1}}, w_{l_{2}}: w_{l_{1}} \notin$ $\left.N_{C H_{t}}\left(w_{l_{2}}\right)\right\}$

3.2.3 A $\stackrel{b}{\rightarrow}$ network: $N_{G_{t+1}}\left(v_{i}\right)$

3.2.4 Each on-line node updates $G_{t}$ by defining $V_{t+1}=V_{t} \cup\left\{v_{i}\right\}, E_{t+1}=E_{t} \cup N_{G_{t+1}}\left(v_{i}\right)$ and $H C_{t+1}=H C_{t} \backslash\left\{\left(v_{j}, v_{k}\right)\right\} \cup\left\{\left(v_{j}, v_{i}\right) \cup\right.$ $\left.\left(v_{i}, v_{k}\right)\right\}$

3.2.5 $A \stackrel{o}{\rightarrow} v_{i}: G_{t+1}$

3.2.6 $\mathrm{A} \stackrel{s}{\longrightarrow} v_{i}: H C_{t+1}$

Output: The supplicant node $S$ becomes a legitimate member of the network.

\section{Access Control}

If a legitimate node $S$ has been off-line or out-of-coverage from stage $t$ and wants to re-enter into the network at stage $r$, its first step should be to contact a legitimate on-line member $A$. Afterwards, $A$ should check whether the period $S$ has been off-line is not greater than $T$. In this case, $S$ has to be authenticated by $A$ through a ZKP based on its knowledge of the secret solution $H C_{t}$ on the graph $G_{t}$.

The aforementioned ZKP begins with the agreement between $A$ and $S$ on the number of iterations $l$ to execute. From there on, in each iteration, $S$ will choose a random permutation $\Pi_{j}\left(V_{t}\right)$ on the vertex set that will be used to build a graph $\Pi\left(G_{t}\right)$ isomorphic to $G_{t}$. The hash value of both the permutation $h\left(\Pi_{j}\left(V_{t}\right)\right)$ and the Hamiltonian cycle in the graph $h\left(\Pi_{j}\left(H C_{t}\right)\right)$ are then sent to $A$. When this information is received by $A$, it chooses a bit $b_{j}$ at random $\left(b_{j} \in \in_{r}\{0,1\}\right)$. Depending on the selected value, $S$ will provide $A$ with the image of the Hamiltonian cycle through the isomorphism, or with the specific definition of the isomorphism. In the verification phase, $A$ will check that the received information was correctly built.

Once the authentication of supplicant $S$ has been successfully carried out, the authenticator $A$ gives him the necessary information to have full access to the protected resources such as the chat application, for example.

Access Control Algorithm

Input: At stage $r$ a supplicant node $S$ that has been off-line since stage $t$ wants to re-enter into the network.

1. $S \rightleftharpoons A$

2. $S \stackrel{o}{\rightarrow} A: G_{t}$ 
3. $A$ checks whether $r-t \leq T$

4. if $r-t>T$ then $S$ is not authenticated

5. otherwise:

- $A \leftrightarrow S: l$

- for $j=1,2, \cdots, l$

$5.1 S$ chooses $\Pi_{j}\left(V_{t}\right)$ and builds $\Pi_{j}\left(G_{t}\right)$ and $\Pi_{j}\left(H C_{t}\right)$, the graph isomorphic to $G_{t}$ and the corresponding Hamiltonian cycle, respectively.

$5.2 S \stackrel{o}{\rightarrow} A:\left\{h\left(\Pi_{j}\left(V_{t}\right)\right), h\left(\Pi_{j}\left(H C_{t}\right)\right)\right\}$

$5.3 A$ chooses the challenge $b_{j} \in \in_{r}\{0,1\}$

$5.4 A \stackrel{o}{\rightarrow} S: b_{j}$

$$
\begin{aligned}
& \text { 5.4.1 If } b_{j}=0 \text { then } \quad S \stackrel{\circ}{\rightarrow} A \quad A \quad \text { : } \\
& \left\{\Pi_{j}\left(G_{t}\right), \Pi_{j}\left(H C_{t}\right)\right\}
\end{aligned}
$$

5.4.2 If $b_{j}=1$ then $S \stackrel{o}{\rightarrow} A: \Pi_{j}$

$5.5 A$ verifies that

a) $\left.\Pi_{j}\left(H C_{t}\right)\right)$ is a valid Hamiltonian cycle in $\Pi_{j}\left(G_{t}\right)$, if $b_{j}=0$

b) the hash function $h$ applied on $\Pi_{j}\left(G_{t}\right)$ coincides with $h\left(\Pi_{j}\left(G_{t}\right)\right)$, if $b_{j}=1$

- if $\exists j \in\{1,2, \ldots, l\}$ such that the verification is negative, then $S$ is isolated.

- otherwise $A \stackrel{s}{\rightarrow} S$ : the necessary information to have full access to protected resources of the network.

Output: Node $S$ is connected on-line to the network.

\section{Proofs of Life}

All on-line legitimate nodes have to confirm their presence in an active way. Such a confirmation is carried out every period of time $T$. It consists in broadcasting a message (proofof-life) to all on-line legitimate nodes.

If some insertion happens during such a period, a proof of life of every on-line legitimate node will be distributed together with the information necessary for the insertion procedure. Otherwise, only the proof of life is required. During such a broadcast every node adds its own proof of life to the broadcast. In this way, when the broadcast reaches the last node, a broadcast back starts containing the proofs of life of all on-line legitimate nodes.

Proof-of-Life Algorithm

Input: At stage $t$ node $A$ is an on-line legitimate node of the network.

1. $A$ initializes its $c l o c k=0$ just after its last proof of life.

2. $\quad$ if clock $>T$ then 2.1

1) $A \stackrel{b}{\leftrightarrow}$ network $: A^{\prime}$ s proof of life

2.1.1 If $A$ receives less than $n / 2$ proofs of life as answers to her broadcast, she stops her proof of life and puts back her clock.

2.1.2 Otherwise: $A \stackrel{b}{\rightarrow}$ network : Received proofs of life

Output: At stage $t+1$ node $A$ continues being an on-line legitimate node of the network of the network.

\section{E. Node Deletion}

The deletion procedure is mainly based on the confirmation of the active presence of on-line legitimate nodes through their proofs of life. Each node should update its stored graph by deleting all those nodes that have not sent any proof of life after a period $T$. This fact implies that each node that has not proven its presence will be deleted from the network, as well as from the Hamiltonian cycle.

Node deletions are explicitly communicated to all on-line legitimate nodes in the second step of broadcasts of proofs of life. This way to proceed allows any node that is off-line in that moment will be able to update its stored graph as soon as it gets access to the network.

Deletion Algorithm

Input: At stage $t$, a node $v_{i}$ is an off-line legitimate node of the network.

1. $A$ initializes her $c l o c k=0$.

2. if clock $>T$ then

$2.1 \forall v_{i} \in V_{t}: A$ checks $v_{i}$ 's proof of life in $A$ 's FIFO queue.

$2.2 A$ updates $V_{t+1}=V_{t} \backslash\left\{v_{i} \in V_{t}\right.$ with no proof \} .

$2.3 A$ updates $E_{t+1}=E_{t} \backslash\left\{\left(v_{i}, v_{j}\right): v_{i} \in V_{t}\right.$ with no proof, $\left.v_{j} \in N_{G_{t}\left(v_{i}\right)}\right\} \cup\left\{\left(v_{j}, v_{k}\right): v_{j}, v_{k} \in\right.$ $\left.N_{H C_{t}\left(v_{i}\right)}\right\}$.

$2.4 A$ updates $H C_{t+1}=H C_{t} \backslash\left\{\left(v_{j}, v_{i}\right),\left(v_{i}, v_{k}\right)\right\} \cup$ $\left(v_{j}, v_{k}\right): v_{i} \in V_{t}$ with no proof, $v_{j}, v_{k} \in$ $N_{H C_{t}\left(v_{i}\right)}$

3. If $A$ started the broadcast used for the $v_{i}$ 's deletion, $A$ adds this information to the second step of the proofof-life broadcast: $A \stackrel{b}{\rightarrow}$ network $: v_{i}$ is deleted.

Output: At stage $t+1$ the node $v_{i}$ has been deleted both from the network and from the graph.

This procedure guarantees a limited growth of the graph that is used in authentication, and at the same time, allows that always the legitimate nodes set corresponds exactly to the vertexes in that graph. Apart from this, it is remarkable the fact that thanks to this procedure the recovery of legitimate members of the network that have been disconnected momentarily is possible.

\section{Assumptions And Security Analysis}

This proposal initially assumes the ideal environment where all legitimate nodes are honest and where no adversary may compromise a legitimate node of the network in order to read its secret stored information. Such assumptions are well suited as a basic model in order to decide under which circumstances the GASMAN is applicable to MANETs. For instance, a possible adaptation of the proposal in order to avoid those hypothesis could be defining a threshold scheme to be used in every step of the GASMAN, so that every proof of life, insertion, access control or deletion operation should be done by a coalition of on-line nodes. Then, a dishonest node would not affect the correct operation of the network. 
It is clear that the proposal inherits some problems of the distributed trust model such as the important necessity that legitimate nodes cooperate. Consequently, it is advisable to include a scheme to stimulate node cooperation.

Finally, another requirement of the GASMAN is the establishment of a secure channel for the insertion procedure. However, that aspect may be easily fulfilled thanks to the fact that most wireless devices are able to communicate with each other via Bluetooth wireless technology.

With respect to possible attacks and due to the lack of a centralized structure, it is natural that possible DOS (Denial Of Service) attacks have as their main objective the chat application. In order to protect the GASMAN against this threat it must be assured that chat messages, although are publicly readable, may be only sent by legitimate on-line members of the network. Another important aspect related to the use of the chat application is the necessary synchronization of the on-line nodes, so a common network clock is necessary. This requirement has been implemented during simulations through the chat application.

MANETs are in general vulnerable to different threats such as identity theft (spoofing) and the man-in-the-middle attack. Such attacks are difficult to prevent in environments where membership and network structure are dynamic and the presence of central directories cannot be assumed. However, our proposal is resistant to spoofing attacks because access control is granted through a ZKP. It implies that any information published through the chat application or sent openly during the execution of access control mechanism becomes useless.

On the other hand, the goal of the man-in-the-middle attack is either to change a sent message or to gain some useful information by one of the intermediate nodes. Again, the use of ZKPs in our protocol implies that reading any transferred information does not reveal any useful information about the secret, so changing the message is not possible since only legitimate nodes whose access has been allowed can use the chat application.

Another active attack that might be especially dangerous in MANETs is the so-called Sybil attack. It happens when a node tries to get and use multiple identities. The most extreme case of this type of attacks is the establishment of a false centralized authority who states the identities of legitimate members. However, this specific attack is not possible against our scheme due to its distributed nature. In the GASMAN, the responsibility of controlling general Sybil attacks will be shared among all the on-line nodes. If an authenticator node detects that a supplicant node is trying to get access to the network by using an ID that is yet being used on-line, such access control must be denied and the corresponding node must be isolated. The same happens when any on-line node detects that an authenticator node is trying to insert a new member into the network with a new ID, and such a node has yet assigned as a vertex ID. Again, such insertion must be denied and the corresponding supplicant node must be isolated. Anyway, if a Sybil attacker enters the network, any of its neighbours will detect it as soon as it sends proofs of life for different vertexes ID.

\section{Performance Analysis}

We now analyze the efficiency of the proposal both from the energy consumption and from computational complexity points of view. We consider the energy consumption which is the result of transmissions of data and processor activities due to authentication tasks. In the proposal there are two phases when computational overhead is more significant: the ZKPbased access control and the periodic checking of stored elements in the FIFO queue. A reduction on the number of rounds of ZKP has a direct effect on the total exchanged messages size in insertions, but a trade-off should be maintained between protocols robustness and performance. Indeed, regarding total data transmission over wireless links, the ZKPs take less than $10 \%$ in a usual situation.

The dominant time-consuming jobs are the periodic proofs of life, which accounts for around $90 \%$ of the total exchanged message size in many cases. However, we found that these compulsory proofs of life imply an incentive technique for stimulating cooperation in authentication tasks. This is due to the fact that nodes that are broadcasters of deletion queries or authenticators in insertions or access controls are exempted from their obligation to broadcast their proofs of life.

In order to reduce data communication cost of the protocol, an increase on the threshold period $T$ might be an option, but again an acceptable balance should be kept. According to our experiments, $T$ should depend directly on the number of legitimate and/or on-line nodes in order to prevent a possible bandwidth overhead in large networks.

For the performance analysis of the proposal we used the Network Simulator NS-2 with the DSR routing protocol. We created several Tcl based NS-2 scripts in order to produce various output trace files that have been used both to do data processing and to visualize the simulation. Within our simulation we used the visualization tool of Network Animator NAM and the NS-2 trace files analyzer of Tracegraph. For the simulation of mobility we used the Setdest program in order to generate movement pattern files using the random Waypoint algorithm.

An example of simulation is shown graphically in Figure 2. Basically, it consists of generating a scenario file that describes the movement pattern of the nodes and a communication file that describes the traffic in the network. These files are used to produce trace files that are analyzed to measure various parameters. An excerpt of the trace files corresponding to the same example is shown in Table I.

The trace files are used to visualize the simulation using NAM, while the measurement values are used as data for plots with Tracegraph. The final graph and Hamiltonian cycle associated to the example network is shown in Figure 3where green is used to indicate the Hamiltonian cycle, blue is used for the inserted nodes and red is used for the edges deleted from the Hamiltonian cycle when inserting new nodes.

In order to study the effectiveness of the GASMAN, we studied it in a set of realistic scenarios. In particular, we 


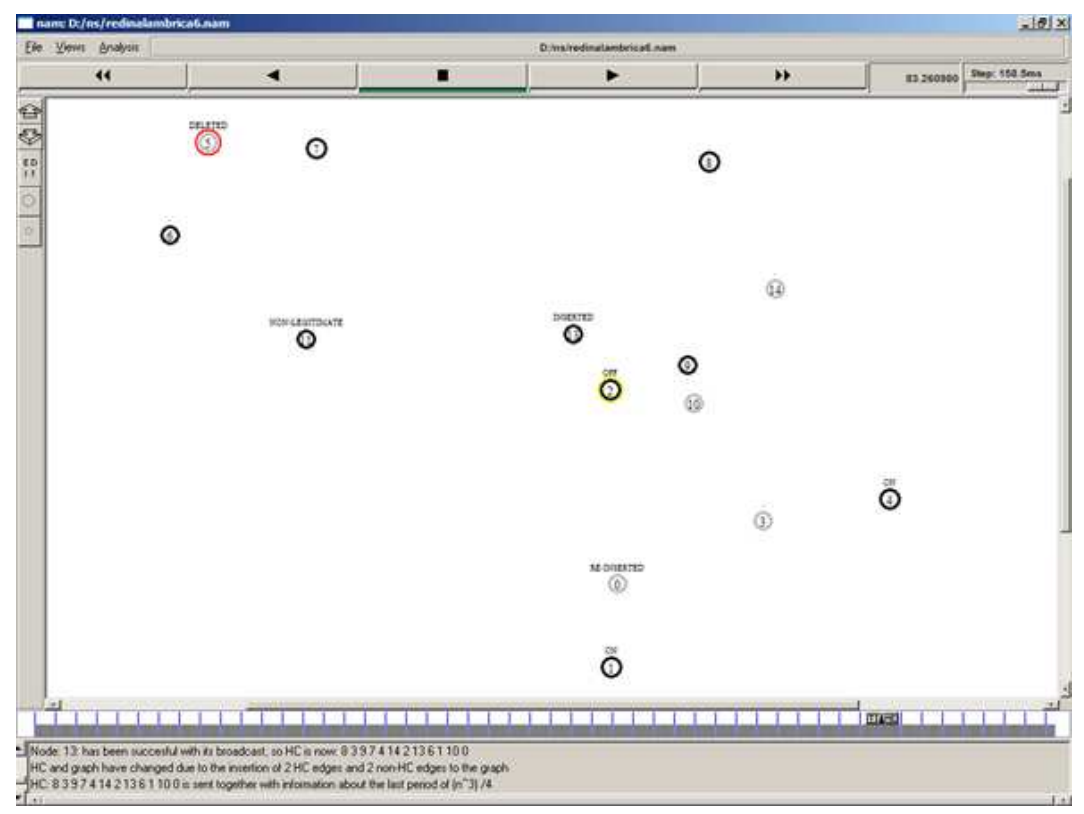

Fig. 2. Example of Network Simulation with NS-2

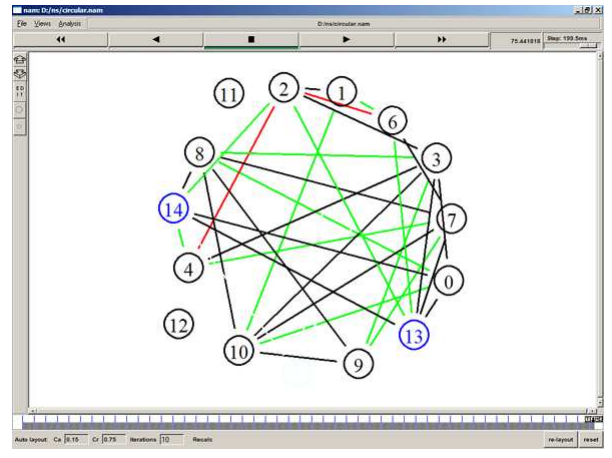

Fig. 3. Example of Final Associated Graph and Hamiltonian Cycle

used the most commonly used mobility model by the research community, the so-called Random Waypoint Model, which uses pause times and random changes in destination and speed.

An extensive number of simulations using NS-2 simulator with 802.11 MAC and DSR routing protocols in order to see the effects of different metrics by varying network density and topology were run. Within the simulations, relationships can be established anytime two nodes are located in close proximity and the random walk mobility model was used with various pause time and maximum speed. In particular, we varied the number of nodes from 15 to 100 . Also, our architecture was evaluated with $250 \times 250,500 \times 500$, and $750 \times 750 \mathrm{~m} 2$ square area of ad-hoc network. In each case, the nodes move around with 0.5 second pause time and $20 \mathrm{~m} / \mathrm{s}$ maximum speeds. The transmission range of the secure channel is 5 meters while that of the data channel is fixed to 250 meters. The period of simulation varied from 60 to 200 seconds. We also changed the probabilities of insertions and deletions in each second from $5 \%$ to $25 \%$, in order to modify the mobility rate and antenna range of nodes from 2 to $15 \mathrm{~m} / \mathrm{s}$ and 100 to 250 meters respectively. This range also defines different frequencies of accesses to the network.

The first conclusions we obtained from the simulations are:

- The protocol scales perfectly to any sort of networks with different levels of topology changes.

- Node density is a key factor for the mean time of insertions, but such a factor is not as big as it might be previously assumed since nodes do not forward two packets of data corresponding to the same proof of life coming from two different nodes.

- A right choice of parameter $T$ should be done according to number of nodes, bandwidth of wireless connections and computation and storing capacities of nodes.

- A positive aspect of the proposal is that the requirements in the devices' hardware are very low.

\section{COnCLusions And Open Questions}

Successful authentication in mobile ad-hoc networks is critical for assuring secure and effective operation of the supported application. This work describes a new authentication scheme, the so-called GASMAN, which was specially designed for 


\begin{tabular}{|c|c|c|}
\hline Time & Event & $\mathrm{HC}$ \\
\hline 0.1 & $0,1,2,3,4,5,6,7,8,9,10$ are legitimate & $8,3,9,7,4,2,6,5,1,10,0$ \\
\hline 1.29 & Insertion of Node 14 is broadcast by Node 4 & $8,3,9,7,4,14,2,6,5,1,10,0$ \\
\hline 1.30 & Nodes $3,1,0$ do not answer to the proof of life & \\
\hline 3.29 & Node 0 reaches 8 and starts a ZKP for re-insertion & \\
\hline 8.69 & Node 3 reaches 4 and starts a ZKP for re-insertion & \\
\hline 9.40 & Node 1 reaches 10 and starts a ZKP for re-insertion & \\
\hline 11.65 & Node 1 turns off & \\
\hline 13.97 & Proof of life started by Node 3 & \\
\hline 14.27 & Nodes 1,2 do not answer to the proof of life & \\
\hline 14.82 & Node 2 reaches 14 and starts a ZKP for re-insertion & \\
\hline 17.27 & Proof of life started by Node 2 & \\
\hline 17.57 & Nodes 1,5 do not answer to the proof of life & \\
\hline 21.71 & Node 5 turns off & \\
\hline 31.40 & Node 1 turns on and Node 2 is chosen for the ZKP & \\
\hline 31.46 & Node 4 turns off & \\
\hline 32.51 & Proof of life started by Node 1 & \\
\hline 32.78 & Nodes $4,5,6$ do not answer to the proof of life & \\
\hline 34.29 & Node 6 reaches 2 and starts a ZKP for re-insertion & \\
\hline 38.51 & Proof of life started by Node 6 & \\
\hline 38.79 & Nodes 4,5 do not answer to the proof of life & \\
\hline 41.46 & Node 1 turns off & \\
\hline 53.25 & Node 1 turns on and Node 0 is chosen for the ZKP & \\
\hline 59.61 & Proof of life started by Node 6 & \\
\hline 59.99 & Nodes 4,5 do not answer to the proof of life & \\
\hline 64.26 & Node 5 is deleted & $8,3,9,7,4,14,2,6,1,10,0$ \\
\hline 64.71 & Node 2 turns off & \\
\hline 72.58 & Node 4 turns on and Node 0 is chosen for the ZKP & \\
\hline 75.41 & Insertion of Node 13 is broadcast by Node 14 & $8,3,9,7,4,14,2,13,6,1,10,0$ \\
\hline 75.43 & Node 2 does not answer to the proof of life & \\
\hline
\end{tabular}

TABLE I

EXAMPLE OF TRACE

MANETs. Such a protocol supports knowledge-based member authentication in server-less environments. The overall goal of the GASMAN has been to design a strong authentication scheme that is able to react and adapt to network topology changes without the necessity of any centralized authority. Its core technique consists of a Zero-Knowledge Proof, in order to avoid the transference of any relevant information. Furthermore, the proposal is balanced since the procedures that the legitimate members of the network have to carry out when the network is updated (insertion or deletion of nodes) imply identical work for every legitimate member of the network.

The development of an initial simulation of the proposal through the NS-2 network simulator has been carried out. The definitive simulation results will be included in a forthcoming version of this work. Also, the study of different applications, practical limitations and possible extensions of the GASMAN may be considered open problems.

\section{REFERENCES}

[1] Aboudagga, N., Tamer, M., Eltoweissy, M., DaSilva, L. and Quisquater,J.J.: Authentication protocols for ad hoc networks: Taxonomy and research issues, Proceedings of the 1st ACM international workshop on Quality of service and security in wireless and mobile networks, October (2005)

[2] Asaeda, H., Rahman, M., Manshaei, H., and Fukuzawa, Y.: Implementation of Group Member Authentication Protocol in Mobile Adhoc Networks, Proceedings of IEEE Wireless Communications and Networking Conference WCNC, Las Vegas, USA April (2006)

[3] Caballero-Gil, P., Hernández-Goya, C.: Strong solutions to the identification problem. Proceedings of COCOON. Lecture Notes in Computer Science Vol. 2108, Springer-Verlag (2001) 257-261
[4] Caballero-Gil, P., Hernández-Goya, C.: Zero-Knowledge Hierarchical Authentication in MANETs. IEICE Transactions on Information and Systems. Letter. E-89-D (2006) 1288-1289

[5] Capkun, S., Buttyan, L., Hubaux, J.P.: Self-organized public-key management for mobile ad-hoc networks. IEEE Transactions on Mobile Computing (2003)

[6] Goldreich, O., Micali, S., Wigderson, A.: How to prove all NPstatements in zero-knowledge, and a methodology of cryptographic protocol design. Proceedings of Crypto '86, Lecture Notes in Computer Science Vol. 263. Springer-Verlag (1986) 171-185

[7] Hahm, S., Jung, Y., Yi, S., Song, Y., Chong, I. and Lim, K.: A SelfOrganized Architecture in Mobile Ad-Hoc Networks. Proceedings of ICOIN, Lecture Notes in Computer Science Vol. 3291 Springer-Verlag (2005) 689-696

[8] Hubaux, J.P., Buttyán, L., Capkun, S.: The quest for security in mobile ad hoc networks. Proceedings of MobiHoc. (2001) 146-155

[9] Jiang, T. and Baras J.S.: Graph Algebraic Interpretation of Trust Establishment in Autonomic Networks. Submitted to Wiley Journal of Networks, May 2005, under review

[10] Maki, S., Aura, T., Hietalathi, M.: Robust membership management for ad-hoc groups. Proceedings of 5th Nordic Workshop on Secure IT Systems NORDSEC (2000)

[11] Saxena, N., Tsudik, G., Yi, J.H.: Efficient node admission for short-lived mobile ad hoc networks. IEEE International Conference on Network Protocols ICNP, November (2005) 269-278

[12] Weimerskirch, A.: Authentication in Ad-hoc and Sensor Networks. $\mathrm{Ph} . \mathrm{D}$. Thesis Ruhr-University Bochum. Germany, July (2004)

[13] Wierzbicki, A., Zwierko, A. and Kotulski, Z.: A New Authentication Protocol for Revocable Anonymity in Ad-Hoc Networks. Proceedings of the IASTED Communication, Network, and Information Security CNIS, Phoenix, AZ, USA (2005)

[14] Wu, H-C., Hwang, M-S. and Liu, C-H.: A Secure Strong-Password Authentication Protocol. Fundamenta Informaticae 68 (2005) 399-406

[15] Zhou, L., Haas, Z.: Securing ad hoc networks. IEEE Networks 13 (1999) 24-30 